\title{
Components of fitness and the PGI polymorphism in the freshwater isopod Asellus aquaticus (L.) 2. Zygotic selection
}

\author{
A. F. Shihab* and \\ D. J. Heath
}

Department of Biology, University of Essex, Colchester, U.K.

* Department of Biology, College of Science, University of Basrah, Basrah, Iraq.

A $2 \frac{1}{2}$ year study of PGI genotypes in a population of the isopod Asellus aquaticus showed consistent seasonal changes in genotype frequencies. In June and July the frequency of the $3 / 4$ heterozygote increased from $0.1-0.25$ while the frequency of the $2 / 3$ ineterozygote decreased from $0 \cdot 15-0 \cdot 05$. Laboratory and field experiments demonstrated that this was due too differential mortality among juveniles, low oxygen concentration being the selective agent.

\section{INTRODUCTION}

Despite 20 years of study the neutralist/selectionist controversy remains unresolved. Spatial variation in allozyme frequencies and their correlation with environmental parameters has little power to discriminate between the two hypotheses (Powers and Place, 1978) because of the unknown historical element. Studies on temporal frequency variation are potentially more informative although the mere demonstration of non-random frequency changes is subject to several interpretations. Firstly differential behaviour of genotypes (Watt, 1983; Dimichele and Powers, 1982) may lead to differential migration and/or biased sampling. If these effects depend on environmental conditions they may cause systematic genotype frequency changes in samples, mimicking the effects of selection. Even if this can be excluded it is not justifiable to assume that the locus under investigation is the target of selection or that the environmental correlate is the selective factor. Linkage between a neutral locus and one affected by selection can cause frequency changes at the neutral locus (Hedrick et al; 1978) and correlation does not imply causation. These effects can only be excluded by a detailed investigation under controlled conditions of the relationship between variation in biochemical properties, physiology and fitness (Hilbish and Koehn, 1985; Watt, 1983; Burton and Feldman, 1983). If this convincingly demonstrated that selection did operate on variation at a particular locus it would be sufficient to refute the neutralist theory for that locus but not to explain how the variation was maintained in the population. Selection may operate at many stages in the life cycle (Christiansen et al., 1973) with no necessary correlation between the performance of a genotype at different stages (Marinkovic and Ayala, 1975) and to fully account for the maintenance of genetic variability requires that net fitnesses of genotypes are estimated. Only certain sorts of organisms are suitable for such an analysis of components of fitness (Lewontin, 1974; Christiansen et al., 1973). Isopods, because they are viviparous, have been used extensively in such studies (Sassaman, 1978; Edwards and Heath, 1983). In the freshwater isopod Asellus aquaticus Verspoor (1983) provided some evidence based on spatial and temporal correlations that temperature and oxygen acted selectively on the PGI polymorphism. The work reported here investigated this in depth by concentrating on temporal variation in PGI genotype frequencies and their relation to population dynamics, survival, fecundity and mating behaviour.

\section{METHODS}

\section{Sampling}

Every month 2 samples of isopods were collected by net from a lake on the University of Essex campus (map reference TM168 031241). The first sample was taken at the end of the second week, the second after four weeks. Individuals were measured (body length), sexed (if over $3 \mathrm{~mm}$ long) 
and electrophoresed to ascertain their PGI genotype. Water temperatures and oxygen concentrations were also taken on each sampling date. $\mathrm{Fu}^{\prime}$ details are given by Shihab (1985).

\section{Experimental}

Two different experimental approaches were used when it became clear from the sampling programme that (a) significant genotypic frequency changes were occurring amongst juveniles in the summer (b) selective mortality related to $\mathrm{O}_{2}$ concentrations and/or temperature was a possible cause.

To discriminate between differential mortality (as opposed to differential migration or differential sampling) samples of animals drawn from the population were confined in closed netting cages in the lake. These cages allowed free movement of water but precluded movement of animals and also effects of differential sampling (since all surviving animals inside the cage were electrophoresed at the end of the experiment). A subsample, electrophoresed before the experiment started, gave an estimate of initial genotype frequencies. On the basis of the time at which frequency changes were observed in the population in 1982 (late June-early August), cages were set up in 1983 and 1984 just before this time. They were inspected at intervals and survivors genotyped when about 50 per cent mortality had occurred. Seven cages were put out each year, each containing 40 juveniles born that May. If selective mortality was the cause of frequency changes in the natural population and if the cage environment was similar to the external environment it was predicted that animals in cages should (a) be reduced in numbers (b) show altered frequencies when compared to the initial subsample.

To identify the selective factor laboratory and field experiments were carried out in 1983 (laboratory) and 1984 (laboratory and field). In the laboratory a large sample of juveniles was divided into 6 groups and each group exposed to low oxygen concentrations at either high or low temperatures ( 3 replicates of each) in 2 litres of lake water. Low oxygen concentrations were produced by boiling the water and then covering the surface with liquid paraffin after the animals had been put in. Under these conditions 50 per cent mortality ensued after 48 hours. Dead animals showed enough enzyme activity to be electrophoresed along with the survivors. Control flasks with normal oxygen concentrations showed no mortality over this period (Shihab, 1985).
These experiments in 1983 showed that oxygen level rather than temperature was the important factor. To investigate this under field conditions an attempt was made to raise the $\mathrm{O}_{2}$ concentration in seven additional cages by aerating them with an electrical air pump. The prediction was that if aeration does raise oxygen levels then aerated and non-aerated cages should show different overall mortality rates and genotype frequencies.

\section{RESULTS}

\section{Frequency changes}

Four alleles segregated at this locus in the population. Electrophoretic analysis of isopods from the Nottingham area where Verspoor sampled suggested that they are the same as the four alleles that he described and are numbered accordingly as 1, 2, 3, 4 (increasing distance from anode). Only 3 genotypes were common and/or showed significant variation and are discussed in this paper; $3 / 3$ (frequency between $0.60-0 \cdot 75), 3 / 4(0 \cdot 10$ $0 \cdot 30)$ and $2 / 3(0 \cdot 05-0 \cdot 20)$. The next most common were $1 / 3(0.01-0.07), 2 / 4(0.01-0.04)$ and $2 / 2$ $(0 \cdot 015)$.

The frequencies of $3 / 4$ and $2 / 3$ are presented in fig. 1. Sample sizes varied between 100 and 260 with a mean size of 182, (Shihab, 1985) giving standard errors of the order of $0 \cdot 05-0.02$. The frequencies showed a striking and complementary pattern of seasonal variation which was similar in all three years and was centred on the May-August period. When the frequencies of these two genotypes were tested individually for heterogeneity within each year (table 1) 5 of the 6 tests showed significant heterogeneity indicating that real changes in frequency were occurring. The data for 3/4 from February 1984 to August 1984 have an associated probability between $0 \cdot 1$ and $0 \cdot 05$. The major features were a sharp decrease in the frequency of $2 / 3$ and a corresponding increase in the frequency of $3 / 4$ in late June and early July followed by an equally sharp reversal in late July and early August. Over the rest of the year they remained relatively constant.

By contrast the frequencies of $3 / 3$ were homogeneous within years (table 1) although there was some evidence of a pattern in fig. 1, with a gradual increase in frequency from April-June and a decrease from June to December. Individual samples from 1982-83 were homogeneous with their counterparts in 1983-84 and can be pooled (Shihab, 1985). The pooled data for the two years 

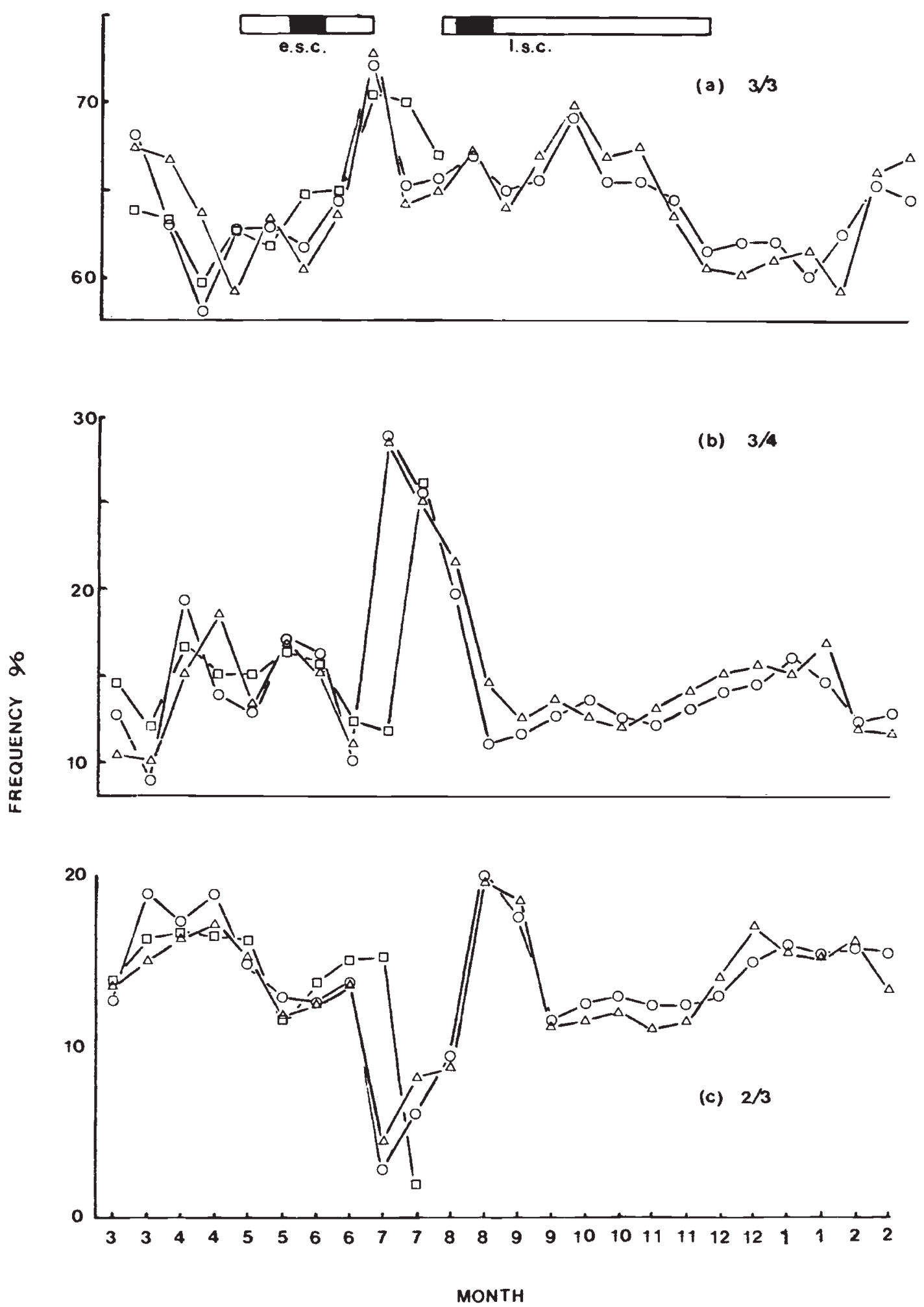

Figure 1 Frequencies of (a) $3 / 3$ (b) $3 / 4$ and (c) 2/3 for the period March 1982-August 1984. $\triangle$ March 1982-February 1983; $\bigcirc$ March 1983-February 1984; $\square$ March 1984-August 1984. e.s.c. $=$ early summer cohort. l.s.c. $=$ late summer cohort. 
Table 1 Heterogeneity $\mathrm{Chi}^{2}$ for the three common genotypes

\begin{tabular}{lllll}
\hline Period & \multicolumn{5}{c}{ Genotype } \\
\hline & $3 / 4$ & $2 / 3$ & $3 / 3$ & df \\
Mar 82-Feb 83 & $51 \cdot 51^{* * *}$ & $37 \cdot 58^{*}$ & $24 \cdot 85$ & 23 \\
Mar 83-Feb 84 & $53 \cdot 87^{* * *}$ & $77 \cdot 39^{* * *}$ & $17 \cdot 18$ & 23 \\
Feb 84-Aug 84 & $17 \cdot 95$ & $20 \cdot 27^{*}$ & $8 \cdot 43$ & 11 \\
\hline
\end{tabular}

${ }^{*} p<0.05,{ }^{* * *} p<0.001$.

then show significant heterogeneity with time $\left(\mathrm{Chi}^{2}=40 \cdot 5\right.$, df 23, p<0.05).

The relation between the frequency changes and the life cycle is also summarised in fig. 1 based on a much more detailed investigation of the population dynamics described by Shihab (1985). The two horizontal rectangles indicate the periods when juveniles entered the population, the smaller filled portion the period when the majority of juveniles appeared. The first period producing the early summer cohort (e.s.c.) when up to $70 \mathrm{per}$ cent of the population were juveniles (Shihab, 1985) was from late April to late June with a peak in late May. The second producing the late summer cohort (l.s.c.) lasted from late July to November with a peak in early August. This cohort was smaller comprising about 15 per cent of the population.

The decline in the frequency of $2 / 3$ and the increase in frequency of $3 / 4$ occurred between these two periods and therefore was not due to differential input of genotypes into the population through natality. The subsequent frequency change coincided with peak production of the late summer cohort.

\section{Environmental variation}

Fig. 2 shows the pattern of seasonal variation in oxygen concentration and temperature. As expected they were negatively correlated with high water temperatures $\left(25^{\circ} \mathrm{C}\right)$ and low oxygen levels (3 ppm) in July and low temperatures/high oxygen in February. The first frequency changes of $2 / 3$ and $3 / 4$ coincided with the highest water temperatures and lowest oxygen levels suggesting that either or both of these factors caused selective mortality. It also suggests that the effect was a threshold effect and that differential mortality only occurred once critical levels have been reached.

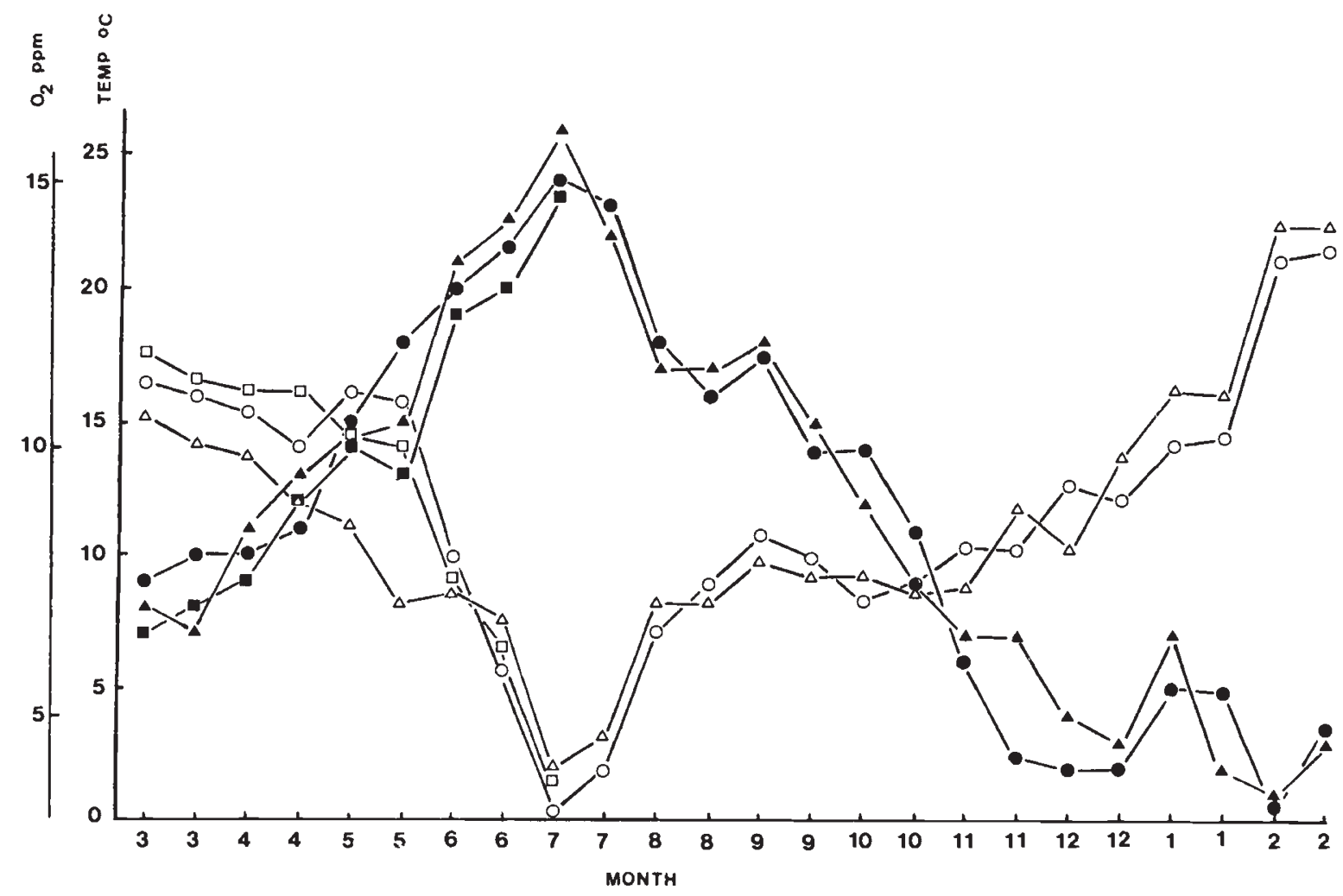

Figure 2 Oxygen concentration (open symbols) and temperature (closed symbols for the period March $1982-J u l y ~ 1984$. $\triangle \Delta$ March 1982-February 1983; OO March 1984-February 1985; $\square$ March 1985-July 1985. 
This hypothesis was tested in the experimental work described in the next section.

\section{Experimental results}

(a) Cages Results for individual cages were homogeneous (Shihab, 1985) and the pooled data are given in table 2(a). They were similar for both years. First mortality has occurred, the numbers being reduced from 280 to 105 and 106 respectively in the two years. Secondly, genotype frequencies amongst the survivors in July were significantly different from the estimated initial frequencies in May, the differences being in the same direction as in the natural population $(2 / 3$ less common, $3 / 4$ more common). Thirdly, frequencies amongst survivors were not significantly different from those in the natural population (uncaged) in July. Frequency changes in the cages must have been due to differential mortality since there was no opportunity for differential migration or biased sampling, no reproduction and the genotypes showed no evidence of mutability (Shihab, 1985). Since changes in the cages closely paralleled those in the field it is reasonable to conclude that these too were the result of differential mortality.

(b) Laboratory Table 2(b) gives the genotype frequencies amongst survivors and in the initial population (obtained by adding frequencies amongst dead animals to those amongst survivors). Data for 1983 and 1984 were homogeneous and have been combined. At both high temperature/low oxygen and low temperature/low oxygen frequencies in survivors were significantly different from initial frequencies. The differences were in the same direction in both cases with $2 / 3$ decreasing, and $3 / 4$ increasing, changes which paralleled (in direction and magnitude) those in the natural population as oxygen concentrations fell. Initial and final oxygen concentrations at high temperature were 2.4 and $1.9 \mathrm{ppm}$ respectively, and for low temperature 2.3 and $1.8 \mathrm{ppm}$. These results clearly demonstrate that low oxygen levels rather than high temperatures caused the selective mortality. The frequency of $3 / 3$ showed no consistent trend while the frequency of others (mainly $1 / 3$ ) showed a decrease.

(c) Aerated cages Table 2(c) gives the genotype frequencies amongst animals in the aerated cages in 1984. There was no mortality and genotype frequencies were not significantly different from those in the May subsample but were significantly different from frequencies amongst survivors in the non-aerated cages $\left(\mathrm{Chi}^{2}=13 \cdot 4, \quad 3 \mathrm{df} p<0 \cdot 01\right)$. Oxygen concentrations in July in the aerated cages ranged from $7.0-6.8 \mathrm{ppm}$,in the non-aerated cages from $4 \cdot 5-3 \cdot 1 \mathrm{ppm}$ and in the water outside the cages from $4 \cdot 7-3 \cdot 1 \mathrm{ppm}$.

Table 2 Results of survival experiments in the laboratory and field cages

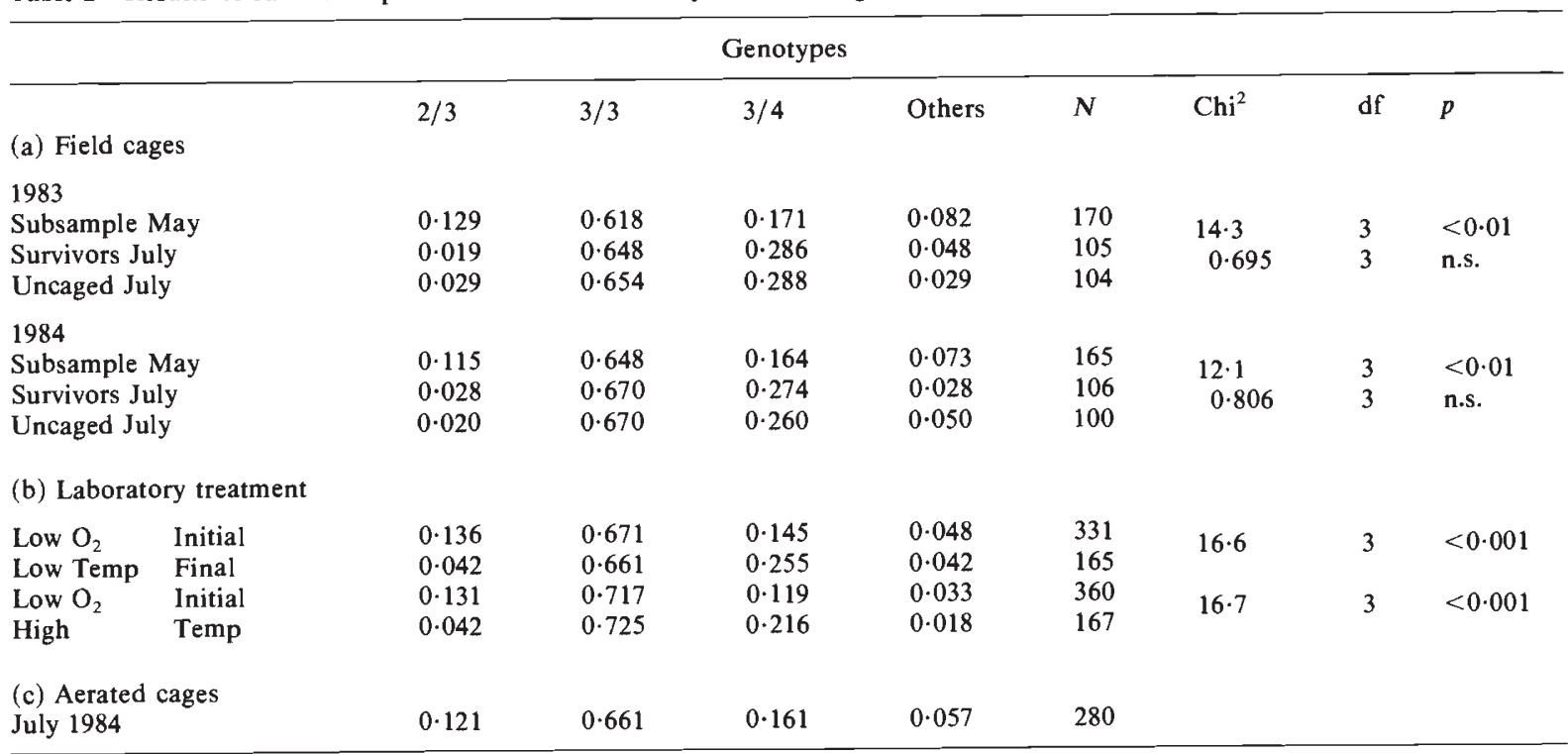


This demonstrates conclusively that in the natural situation it was the low oxygen concentrations that (a) caused mortality (b) favoured $3 / 4$ compared to $2 / 3$.

\section{DISCUSSION}

These results show that the genetic make up of the population with respect to variation at the PGI locus (or more strictly that section of the chromosome marked by the PGI locus) showed systematic seasonal changes due to selective mortality. Although selective mortality (zygotic selection) has often been inferred for allozyme variants (e.g. Christiansen et al., 1973; Corbin, 1977; Hilbish and Koehn, 1985; Sassaman, 1978; Watt, 1983) it has only been rigorously demonstrated in a few instances (e.g. Burton and Feldman, 1985; Johnson, 1971).

The environmental factor responsible has been identified clearly as low oxygen concentration rather than high temperature although temperature has been most frequently implicated as a cause of PGI allele frequency variation. Correlations between temperature and geographic frequency variation have been described in the butterfly Colias eurytheme (Watt, 1983) in the sea anemone Metridium senile (Hoffman, 1981a), the fish Fundulus heteroclitus (Powers and Place, 1978) and in the mussel Mytilus edulis (Koehn et al., 1984). In vitro tests of the biochemical activity of PGI allozymes have shown that there are temperature related differences between variants in Metridium (Hoffman, 1981b), Mytilus (Hall, 1985) and Colias (Watt, 1983) which can be related plausibly to fitness and can provide partial explanations for the observed geographic distributions of the alleles. Although it has not been shown that variation at the PGI locus in Asellus is the target of selection, there are possible mechanisms by which low oxygen concentration could act selectively on PGI genotypes. Under hypoxia Asellus may shift to anaerobic respiration leading to the accumulation of lactic acid and changes in intracellular $\mathrm{pH}$. If the variants have different $\mathrm{pH}$ requirements this could translate into differences in survival. An alternative mechanism involves the inhibition of PGI by citrate (Shihab, 1985).

In August the frequency changes were reversed; frequencies of $2 / 3$ rose rapidly while those of $3 / 4$ fell. The cause is not clear but it seems unlikely to be selective mortality due to oxygen and temperature levels favourable to $2 / 3$. If this was the case we would expect to have seen a rise in the frequency of $2 / 3$ earlier in the summer (May/June) when oxygen and temperature levels were the same as they were in August. This did not occur. In addition the situation in both May/June and August will be complicated by any differences between genotypes in reproductive output. Shihab and Heath (1986) have shown that the mean number of young produced by $2 / 3$ and $3 / 4$ were (respectively) 65 per cent and 40 per cent that of $3 / 3$. In addition there was some evidence that $2 / 2$ was under-represented in females producing the e.s.c. and over-represented in females producing the l.s.c. Amongst the mating animals, pairing appeared to be at random among the 3 genotypes considered here (Shihab, 1985). This additional information allows a further interpretation of the frequency changes. During the production of the e.s.c. the frequency of $3 / 3$ rises as would be predicted on the basis of its higher fecundity. A test for linear trend in proportions (Snedecor and Cochran, 1967) on the frequency of $3 / 3$ during the period of production of the e.s.c. shows a significant increase in 1982 and 1983 (Shihab, 1985). This increase was predicted on the basis of the high reproductive output of $3 / 3$ and was compensated for by decreases in the frequency of $2 / 3$ and $3 / 4$ caused by their lower reproductive output. In the case of the frequency of $2 / 3$ this decrease would be exacerbated if the $2 / 2$ homozygote is under-represented among mating animals, because most mating of $2 / 2$ would be with $3 / 3$.

After the selective mortality described in this paper occurred there was a further input into the population by reproduction. Fewer animals contribute to the production of the 1.s.c. and brood sizes are smaller but the differences in reproductive output between genotypes persist (Shihab and Heath, 1986). 2/3, because of its higher fecundity relative to $3 / 4$, should increase in frequency, this rise being further enhanced by the tendency of $2 / 2$ (mating predominantly with $3 / 3$ ) to be overrepresented among the parents of the 1.s.c. This would account for the frequency reversal of $2 / 3$ and $3 / 4$ observed in July/ August.

Although these data on survival and reproductive output account satisfactorily for some of the frequency changes they do not account for the stability of the polymorphism especially in relation to the frequency of $3 / 3$. This genotype appears to have a reproductive advantage and no detectable disadvantage under summer conditions of low oxygen concentration or in mating (Shihab, 1985) Its frequency would be expected to rise from year to year, yet this does not appear to have happened over the 3 years studied. However, fig. $1 a$ suggests 
that from a maximum frequency of 70 per cent at the end of June the frequency of $3 / 3$ declined over late summer and autumn to a frequency of 60 per cent in January (compensated for by increases in frequency of $2 / 3$ and $3 / 4$ ). This could be accounted for by lower winter survival of $3 / 3$ but there is no direct evidence for this.

\section{REFERENCES}

BURton, R. S. AND FELdman, M. w. 1983. Physiological effects of an allozyme polymorphism: glutamate-pyruvate transaminase and response to hyperosmotic stress in the copepod Tigriopus californicus. Biochem. Genet., 21, 239-251.

CHRISTIANSEN, F. B. FRYDENBERG, O. AND SIMONSEN, V. 1973. Genetics of Zoarces populations. IV. Selection component analysis of an esterase polymorphism using population samples including mother-offspring combinations. Hereditas, 73, 291-304.

CORBIN, K. W. 1977. Phosphoglucose isomerase polymorphjism and natural selection in the sand crab Emerita talpoida. Evolution, 31, 331-340.

Dimichele, L. AND POWERS, D. A. 1982. Physiological basis for swimming endurance differences between LDH-B genotypes of Fundulus heteroclitus. Science, 216, 1014-1016.

EDWARDS, J. P. AND HEATH, D. J. 1983. Dynamics of an enzyme polymorphism in the isopod. Sphaeroma rugicauda (Leach). II. Sexual, gametic and fecundity selection. Heredity, $51,477-486$.

HALL, J. G. 1985.. Temperature related kinetic differentiation of glucose phosphate isomerase alleloenzymes isolated from the blue mussel, Mytilus edulis. Biochem. Genet., 23, 705-729.
HEDRICK, P., JAIN, S. AND HOLDEN, L. 1978. Multilocus systems in evolution. Evol. Biol., 11, 101-184.

HILBISH, T. J. AND KOEHN, R. K. 1985. The physiological basis of natural selection at the Lap locus. Evolution, 39, 1309-1317.

HOFFMAN, R. J. 1981a. Evolutionary genetics of Metridium senile. II. Geographic patterns of allozyme variation. Biochem. Genet., 19, 145-154.

HOFFMAN, R. J. 1981 b. Evolutionary genetics of Metridium senile. I. Kinetic differences in phosphoglucose isomerase allozymes. Biochem. Genet., 19, 129-144.

JOHNSON, M. S. 1971. Adaptive lactate dehydrogenase variation in the crested blenny, Anoplarchus. Heredity, 27, 205-226.

LEWONTIN, R. C. 1974. The Genetic Basis of Evolutionary Change, Columbia University Press, New York.

MARINKOVIC, D. AND AYALA, F. J. 1975. Fitness of allozyme variants in Drosophila pseudoobscura. II. Selection at the Est-5, Odh and Mdh-2 loci Genet. Res., 24, 137-149.

POWERS, D. A. AND PLACE, 9A. R. 1978. Biochemical genetics of Fundulus heteroclitus (L). I. Temporal and spatial variation in gene frequencies of $L d h-\mathrm{B}, M d h-\mathrm{A}$ and $P g m-\mathrm{A}$. Biochem. Genet., 16, 593-607.

SASSAMAN, C. 1978. Dynamics of a lactate dehydrogenase polymorphism in the woodlouse Porcellio scaber Latr: Evidence for partial assortative mating and heterosis in natural populations. Genetics, 88, 591-609.

SNEDECOR, G. W. AND COCHRAN, W. G. 1967. Statistical methods The Iowa State University Press, Iowa.

SHIHAB, A. 1985. Dynamics of an enzyme polymorphism in the isopod Asellus aquaticus. Ph.D. Thesis, University of Essex.

VERSPOOR, E. 1983. Allozyme frequencies in Western European populations of Asellus aquaticus (L.) Isopoda and their association with water pollution. Biol. J. Linn. Soc., 19, 275-293.

WATT, w. B. 1983. Adaptation at specific loci II. Demographic and biochemical elements in the maintenance of the Colias PGI polymorphism. Genetics, 103, 691-724. 